Research Article

\title{
Regularization of the Shock Wave Solution to the Riemann Problem for the Relativistic Burgers Equation
}

\author{
Ting Zhang and Chun Shen \\ School of Mathematics and Statistics Science, Ludong University, Yantai, Shandong 264025, China \\ Correspondence should be addressed to Chun Shen; shenchun3641@sina.com
}

Received 25 June 2014; Accepted 10 August 2014; Published 20 August 2014

Academic Editor: Milan Pokorny

Copyright ( 2014 T. Zhang and C. Shen. This is an open access article distributed under the Creative Commons Attribution License, which permits unrestricted use, distribution, and reproduction in any medium, provided the original work is properly cited.

The regularization of the shock wave solution to the Riemann problem for the relativistic Burgers equation is considered. For Riemann initial data consisting of a single decreasing jump, we find that the regularization of nonlinear convective term cannot capture the correct shock wave solution. In order to overcome it, we consider a new regularization technique called the observable divergence method introduced by Mohseni and discover that it can capture the correct shock wave solution. In addition, we take the Helmholtz filter for the fully explicit computation.

\section{Introduction}

The Euler and Navier-Stokes equations are well known as the fundamental laws governing fluid dynamics which still have many challenges needed to be overcome. One reason lies in that the nonlinear terms in the equations give rise to small scale structures in the form of shock and turbulence. Thus it is hoped that an efficient method should be created to capture the small scale structures. The regularization idea through convoluting some terms with the filter introduced by Chen et al. [1-3] and Cheskidov et al. [4] has been well used to deal with the above problems, which belongs to the class of models known as the $\alpha$-model. In fact, it is similar to the Leray idea [5] by using convolution to deal with the incompressible Navier-Stokes equations and thus is usually named the Leraytype regularization in the literature.

The $\alpha$-type regularization in the context of various hydrodynamic models has been widely considered, for instance in [6-12]. The $\alpha$-type regularization technique has also been extended to both the Euler equations [13-15] and the Navier-Stokes equations $[4,9,10,16-19]$. In addition, the Lagrangian averaging approach has also been adopted to capture the shock and turbulence for the Euler and NavierStokes equations by using a filtered convective velocity in the nonlinear terms, such as in $[4,15,20,21]$. Recently, the $\alpha$-type regularization on hyperbolic systems of conservation laws has also been carried out such as in [22-24].

The inviscid Burgers equation

$$
u_{t}+\left(\frac{u^{2}}{2}\right)_{x}=0
$$

is an important toy model in computational fluid dynamics. It is also the simplest example of nonlinear scalar conservation laws and often serves as a model for more complicated equations due to the fact that it shares the same nonlinear convective term as the Euler and Navier-Stokes equations. It is well known that the shock wave may appear in the solution to (1) even for smooth initial data. Classically, the Burgers equation (1) was regularized with a dissipative term such as viscosity [25]. In addition, another well-known example of the regularization to (1) is to add the linear dispersion term which results in the KdV equations [26].

A filtered convective velocity in the nonlinear term of Burgers equation as an alternative regularization technique

$$
\begin{gathered}
u_{t}+\bar{u} u_{x}=0, \\
\bar{u}(x, t)=\psi_{\alpha}(x) * u(x, t)
\end{gathered}
$$

has been introduced in [27], in which the convolution is only in the $x$ variable, $\psi_{\alpha}(x)=(1 / \alpha) \psi(x / \alpha)$, and $\psi(x)$ is 
a smoothing kernel and usually is assumed to be a smooth, even, integrable function normalized to have total integral equal to one. In particular, the Helmholtz filter is the most important one for its convenient inversion techniques. With this regularization, the convective velocity in the Burgers equation in (1) is replaced with a filtered velocity in the convectively filtered Burgers (abbrreviated as CFB) equation (2). Recently, the Leray regularization technique used on the Burgers equation (1) has been extensively studied such as in $[14,28-30]$. It has been shown that the existence and uniqueness of the solutions to the CFB equation (2) have been considered by Norgard and Mohseni $[14,30]$. It was proved in [30] that the solutions to the CFB equation (2) converge to the entropy solution of the inviscid Burgers equation (1) under certain initial conditions. In addition, Bhat and Fetecau [28] have studied the Riemann problem for the CFB equation (2) and discovered that the Leray regularization technique captures the correct shock solution of the inviscid Burgers equation (1) as $\alpha \rightarrow 0$.

In this note, we consider the Leray regularization technique on the following relativistic Burgers equation:

$$
u_{t}+\left(\frac{\sqrt{1+\varepsilon^{2} u^{2}}-1}{\varepsilon^{2}}\right)_{x}=0
$$

where $\varepsilon$ denotes the inverse of the normalized speed of light. It is derived in [31] from the Euler system of relativistic compressible flows in a curved space time when all the geometric effects are neglected. In addition, it also retains several key features of the relativistic Euler equations. It is clear to see that the flux function $f(u)=\left(\sqrt{1+\varepsilon^{2} u^{2}}-\right.$ $1) / \varepsilon^{2}$ is strictly convex and then the conservation law is genuinely nonlinear. Furthermore, its characteristic value, namely, the convective velocity, is $\lambda=f^{\prime}(u)=u / \sqrt{1+\varepsilon^{2} u^{2}}$. Formally, one recovers the inviscid Burgers equation (1) in the nonrelativistic limit $\varepsilon \rightarrow 0$ in (4). At first, we want to consider the regularization of the convective velocity for the relativistic Burgers equation (4) and confine ourselves to the Riemann initial data only; namely, we consider the Riemann problem for the convectively filtered relativistic Burgers (abbreviated as CFRB) equation

$$
u_{t}+\overline{\left(\frac{u}{\sqrt{1+\varepsilon^{2} u^{2}}}\right)} \cdot u_{x}=0
$$

with the Riemann initial data

$$
u(x, 0)= \begin{cases}u_{l}, & x<0 \\ u_{r}, & x>0\end{cases}
$$

Like the method for the CFB equation (2) in [28], we can also solve the Riemann problem for the CFRB equation (5) by using the method of characteristics. In this note, we are concerned with the shock capturing capabilities of the regularization technique. Thus, we draw our attention to the case $u_{l}>u_{r}$ in which the shock wave appears in the Riemann solution to (4) and (6). However, we discover that this regularization is not a reasonable means to capture the formation of shock wave due to the fact that the limits $\alpha \rightarrow 0$ of the Reimann solutions to (5) and (6) do not converge to the corresponding ones to (2) and (6) owing to the differential shock speeds when $u_{l}>u_{r}$.

Thus, we can see that arbitrarily applying the regularization technique does not lead to a satisfactory result, even for a scalar conservation law [24] such as the relativistic Burgers equation. In order to remedy it, we need to search for a reasonable regularization of equation by means of filtering variables. Inspired by the recent work by Mohseni [32], Norgard and Mohseni [15], and Villavert and Mohseni [23] we consider a new regularization technique called the observable divergence method to the relativistic Burgers equation (4) as

$$
u_{t}+\overline{\left(\frac{f(u)}{u}\right)} \cdot u_{x}+\bar{u} \cdot\left(\frac{f(u)}{u}\right)_{x}=0
$$

namely

$$
u_{t}+\overline{\left(\frac{u}{\sqrt{1+\varepsilon^{2} u^{2}}+1}\right)} \cdot u_{x}+\bar{u} \cdot\left(\frac{u}{\sqrt{1+\varepsilon^{2} u^{2}}+1}\right)_{x}=0
$$

which is named the the observable relativistic Burgers (abbreviated as $\mathrm{ORB}$ ) equation in this note.

The observable divergence method has been confirmed to be an effective method for the one-dimensional homentropic Euler and full Euler equations in [15]. Unlike the aforementioned Leray-type regularization technique, the observable divergence method can capture correctly the entropy solutions for the shock-tube problem and appears to be a valid shock regularization for the gas dynamic equations. Motivated by such work, we make a further step to consider the Riemann problem for the ORB equation (8) in detail. We discover that it shares the same Riemann solution to the relativistic Burgers equation (4) when $u_{l}>u_{r}$, which implies that the Riemann solution to (8) and (6) has no dependence on the regularization parameter $\alpha$. It is clear that the Riemann solution to (8) and (6) converges to the corresponding Riemann solution to (4) and (6) as $\alpha \rightarrow$ 0 . Thus the observable divergence method can capture the shock wave solution correctly for the relativistic Burgers equation (4). In addition, due to the special structure of the Burgers equation (1), we notice the fact that we can obtain the same form (2) when we regularize Burgers equation (1) by using the regularization of the convective velocity or the observable divergence method. Maybe this is the reason why CFB equation (2) can capture the correct shock wave solution.

The different regularization methods have been extensively used to study the motion of large eddies in a turbulent flow, which usually adopt the approximation of local and spacial averages in a turbulent flow, such as the Bardina model and an approximate deconvolution model. The Bardina model was proposed by Bardina et al. [33] by taking the mean of the Navier-Stokes equations and then neglecting the residual stress and its variations. In addition, the simplified Bardina model was also proposed by Layton and Lewandowski [34] and the existence and uniqueness of strong solutions were obtained therein. It is worthwhile to notice 
that an approximate deconvolution model (abbreviated as ADM) was proposed by Stolz and Adams [35] to study the large-eddy simulation through employing an approximate deconvolution of a filtered quantity by repeated filtering. The main ingredient of ADM is to approximate the nonfiltered field by using the truncated series expansion of the inverse filter operator. We can also see [36] for the comparison among the three $\alpha$-models known as the LANS- $\alpha$, Leray- $\alpha$, and Bardina models and [37] for the comparison between the approximate deconvolution model and the Bardina model. Compared with the above regularization methods, to study the motion of large eddies in a turbulent flow, the regularization method in this paper is expected to capture the correct shock wave speed for hyperbolic conservation laws.

The paper is organized as follows. In Section 2, we deliver the Riemann solution the relativistic Burgers equation (4) and then depict the Leray-type regularization with the Helmholtz mollifier. In Section 3, we consider the Riemann problem for CFRB equation (5) and explain that the limit of Riemann solution to (5) and (6) cannot converge to the corresponding one to (4) and (6) as $\alpha \rightarrow 0$. In Section 4 , we consider the Riemann problem for the ORB equation (8) and discover that it has the same Riemann solution as the relativistic Burgers equation (4) when $u_{l}>u_{r}$. Moreover, we prove rigorously that the limit of Riemann solution to (5) and (6) converges to the corresponding one to (8) and (6) as $\alpha \rightarrow 0$. Finally, the discussion is carried out in Section 5.

\section{Preliminaries}

In this section, we first give some results on the Riemann problem to the relativistic Burgers equation (4). It is noticed that (4) is a special scalar conservation law with the convex flux function, and thus it is well known how to solve the Riemann problem (4) and (6). Obviously, it has only one eigenvalue $\lambda=f^{\prime}(u)=u / \sqrt{1+\varepsilon^{2} u^{2}}$ and thus it is genuinely nonlinear provided that $u \neq 0$.

For the case $u_{l}<u_{r}$, the Riemann solution consists of a rarefaction wave which can be expressed as

$$
u(x, t)= \begin{cases}u_{l}, & x<f^{\prime}\left(u_{l}\right) t, \\ \frac{\xi}{\sqrt{1-\varepsilon^{2} \xi^{2}}}, & f^{\prime}\left(u_{l}\right) t \leq x \leq f^{\prime}\left(u_{r}\right) t, \\ u_{r}, & x>f^{\prime}\left(u_{r}\right) t,\end{cases}
$$

where $\xi=x / t$. For the case $u_{l}>u_{r}$, the Riemann solution is a shock wave which can be expressed as

$$
u(x, t)= \begin{cases}u_{l}, & x<\sigma t, \\ u_{r}, & x>\sigma t,\end{cases}
$$

in which $\sigma$ is the shock propagation speed and can be obtained from the Rankine-Hugoniot condition of (4) as

$$
\sigma=\frac{u_{l}+u_{r}}{\sqrt{1+\varepsilon^{2} u_{l}^{2}}+\sqrt{1+\varepsilon^{2} u_{r}^{2}}} .
$$

Thus, it is obvious to see that the limit of Riemann solution to the relativistic Burgers equation (4) and (6) converges to the corresponding Riemann solution to the Burgers equation (1) and (6) in the nonrelativistic limit $\varepsilon \rightarrow 0$ (namely, the speed of light tends to infinity).

Now, we simply describe the acceptable filters used in averaging the chosen quantities in the equations; see [14, 28] for example. Several assumptions on the averaging kernel are made, such as the fact that it is required to be a nonnegative smooth and even function. Usually, we need also to assume that it is nonincreasing with respect to the absolute value of the argument and integrable normalized to have total integral equal to one. For simplicity, the Helmholtz mollifier

$$
\psi(x)=\frac{1}{2} \exp (-|x|)
$$

is chosen here which is an averaging kernel of special interest for its convenient inversion techniques. It is remarkable that $\psi(x)$ is not derivative at $x=0$. Furthermore, the kernel should be equipped with a small parameter $\alpha>0$ which acts as a scaling of the kernel and controls the level of filtering, which can be expressed as

$$
\psi_{\alpha}(x)=\frac{1}{\alpha} \psi\left(\frac{x}{\alpha}\right)=\frac{1}{2 \alpha} \exp \left(-\left|\frac{x}{\alpha}\right|\right) .
$$

For a given real-valued function $f$, we can use the Helmholtz mollifier (13) to define the filter of $f(x)$ by the convolution operator which can be represented by a bar as

$$
\bar{f}(x)=\int_{-\infty}^{+\infty} f(x-y) \psi_{\alpha}(y) d y,
$$

which satisfies

$$
f(x)=\bar{f}(x)-\alpha^{2} \Delta \bar{f}(x) .
$$

It is clear to see that the averaging kernel in (13) converges to the Dirac delta function and consequently the filter $\bar{f}(x)$ in (14) converges to $f(x)$ as $\alpha \rightarrow 0$.

Finally, let us simply discuss the observable divergence method, such as the fact that we can see [15] for more details. This method is based on a conservation law perspective and addresses the cascade of energy generated by the nonlinear terms. Suppose that there is single or multiple conservation laws which can be written as

$$
Q_{t}+(u Q)_{x}=0
$$

The observable divergence method is to expand the nonlinear flux term by using the product rule and then apply a filter to the differentiated quantities. Through the above process, it leads to

$$
Q_{t}+\bar{u} Q_{x}+\bar{Q} u_{x}=0
$$

In particular, if we adopt Helmholtz mollifier (13), then (17) can be rewritten in the conservative form

$$
Q_{t}+\left(\bar{u} Q+\bar{Q} u-\bar{u} \bar{Q}+\alpha^{2} \bar{u}_{x} \bar{Q}_{x}\right)_{x}=0,
$$

in which (15) has been used. 


\section{Riemann Problem for CFRB Equation (5)}

In this section, we are mainly concerned with the Riemann problem for the CFRB equation (5). In order to obtain the exact solution of Riemann problem (5) and (6), let us fix $\psi$ to be the Helmholtz filter (13). Based on the explicit expression, we make a further step to discuss the limit of the Riemann solution for (5) and (6) as $\alpha \rightarrow 0$ and compare the result with the corresponding Riemann solution for the relativistic Burgers equation (4) and (6). Let us draw our attention to the case $u_{l}>u_{r}$ due to the fact that the shock wave appears in the Riemann solution to (4) and (6). The Riemann problem for the CFB equation (2) has been considered in [28] where the technique of Leray-type regularization is a reasonable means of capturing shock formation. The related discussion on this topic was also carried out in [24] for scalar conservation law where the technique of Leray-type regularization cannot capture the correct shock wave solution and thus it is unsuitable as a regularization for the general scalar conservation law. Here we take relativistic Burgers equation (4) by using the Helmholtz filter (13) for the Leraytype regularization, which enables us to obtain a fully explicit expression of the trajectories and solutions.

Local and global existence can be obtained by applying the method of characteristic, which is similar to the process for CFB equation (2) in [28]. The particle paths are defined by the trajectories $\eta(X, t)$ which emit from the position $X$ at the time $t=0$ and obey

$$
\frac{d \eta}{d t}(X, t)=\overline{f^{\prime}(u)}=\overline{\left(\frac{u}{\sqrt{1+\varepsilon^{2} u^{2}}}\right)}
$$

which is the corresponding characteristic equations for CFRB equation (5). The solution $u$ is a constant along the particle path such that we have $u(\eta(X, t), t)=u_{0}(X)$ for all $t$. More precisely, (19) can be rewritten as

$$
\frac{d \eta}{d t}(X, t)=\int_{-\infty}^{+\infty} \frac{\psi_{\alpha}(\eta(X, t)-y) \cdot u(y, t)}{\sqrt{1+\varepsilon^{2} u^{2}(y, t)}} d y
$$

through the change of variables $y=\eta(Y, t)$, which becomes

$$
\begin{aligned}
& \frac{d \eta}{d t}(X, t) \\
& \quad=\int_{-\infty}^{+\infty} \frac{\psi_{\alpha}(\eta(X, t)-\eta(Y, t)) \cdot u_{0}(Y) \cdot \eta_{Y}(Y, t)}{\sqrt{1+\varepsilon^{2} u_{0}^{2}(Y)}} d Y .
\end{aligned}
$$

In particular, if we choose Helmholtz mollifier (13) and consider the Riemann initial data (6), then (21) turns into

$$
\begin{aligned}
& \frac{d \eta}{d t}(X, t) \\
& =\int_{-\infty}^{0} \frac{1}{2 \alpha} \cdot \exp \left(-\frac{|\eta(X, t)-\eta(Y, t)|}{\alpha}\right) \cdot \frac{u_{l} \cdot \eta_{Y}(Y, t)}{\sqrt{1+\varepsilon^{2} u_{l}^{2}}} d Y \\
& +\int_{0}^{+\infty} \frac{1}{2 \alpha} \cdot \exp \left(-\frac{|\eta(X, t)-\eta(Y, t)|}{\alpha}\right) \cdot \frac{u_{r} \cdot \eta_{Y}(Y, t)}{\sqrt{1+\varepsilon^{2} u_{r}^{2}}} d Y .
\end{aligned}
$$

One can obtain the explicit expression of the particle paths to Riemann problem (5) and (6) with a similar calculation as in $[24,28]$ as follows:

$$
\begin{aligned}
& \frac{d \eta}{d t}(X, t)
\end{aligned}
$$

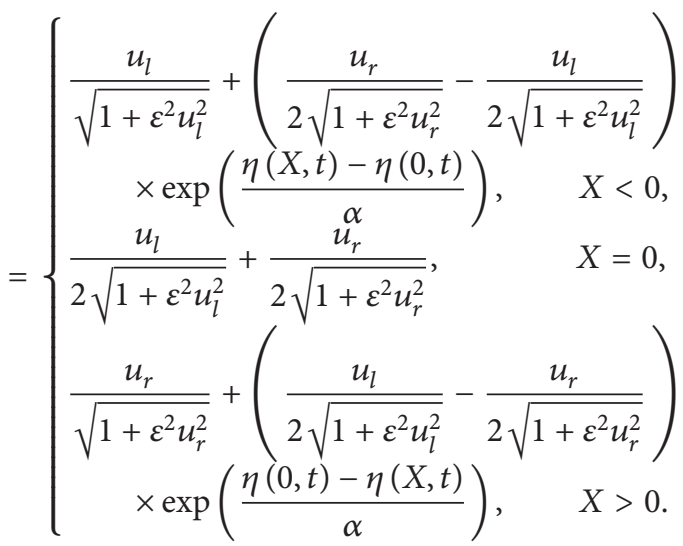

Let us denote

$$
\tau=\frac{u_{l}}{2 \sqrt{1+\varepsilon^{2} u_{l}^{2}}}+\frac{u_{r}}{2 \sqrt{1+\varepsilon^{2} u_{r}^{2}}}
$$

with the initial condition $\eta(0,0)=0$ in mind; then it follows directly from (23) that $\eta(0, t)=\tau t$, which implies that the trajectory emitting from the initial discontinuous point $X=0$ is a line of slope $\tau$ which is exactly the shock speed to the Riemann problem for CFRB equation (5). Let us also denote

$$
\theta=\frac{u_{r}}{2 \sqrt{1+\varepsilon^{2} u_{r}^{2}}}-\frac{u_{l}}{2 \sqrt{1+\varepsilon^{2} u_{l}^{2}}}
$$

then it is easy to get $\theta<0$ for $u_{l}>u_{r}$ since $f^{\prime}(u)=$ $u / \sqrt{1+\varepsilon^{2} u^{2}}$ is monotonically increasing with respect to $u$. 
Noticing the initial condition $\eta(X, 0)=X$, the trajectories $\eta(X, t)$ to the Riemann problem (5) and (6) are given by

$$
\begin{aligned}
& \eta(X, t) \\
& =\left\{\begin{array}{rlrl}
\tau t-\alpha \ln [1+ & (-1 \\
& \left.\left.+\exp \left(-\frac{X}{\alpha}\right)\right) \cdot \exp \left(\frac{\theta t}{\alpha}\right)\right], & & X<0, \\
\tau t, & & X=0, \\
\tau t+\alpha \ln [1+(-1 & & \\
& \left.\left.+\exp \left(\frac{X}{\alpha}\right)\right) \cdot \exp \left(\frac{\theta t}{\alpha}\right)\right], & & X>0 .
\end{array}\right.
\end{aligned}
$$

It follows directly from $(26)$ that $(\partial \eta / \partial X)(X, t)>0$ for any fixed $t$ and $\alpha$ and thus $\eta(X, t)$ is monotonically increasing with respect to $X$ which implies that the characteristic curves cannot intersect in finite time. Furthermore, we have $\lim _{t \rightarrow+\infty}(\eta(X, t)-\tau t)=0$ for any given $X$ and $\alpha$, which implies that all the characteristic curves take the line $x=\tau t$ as their asymptote and cannot pass through it. In addition, we let $X$ and $t$ be fixed and take the limit $\alpha \rightarrow 0$. If $X<0$, then we have

$$
\lim _{\alpha \rightarrow 0} \eta(X, t)= \begin{cases}X+\frac{u_{l} t}{\sqrt{1+\varepsilon^{2} u_{l}^{2}},} & t<\frac{X}{\theta}, \\ \tau t, & t>\frac{X}{\theta},\end{cases}
$$

which implies that the limit trajectory starting from the initial point $X$ follows the line of slope $u_{l} / \sqrt{1+\varepsilon^{2} u_{l}^{2}}$ until it meets the shock; consequently it follows along the shock line $x=\tau t$ after this time. Similarly, if $X>0$, then we also have

$$
\lim _{\alpha \rightarrow 0} \eta(X, t)= \begin{cases}X+\frac{u_{r} t}{\sqrt{1+\varepsilon^{2} u_{r}^{2}}}, & t<-\frac{X}{\theta}, \\ \tau t, & t>-\frac{X}{\theta} .\end{cases}
$$

The values of $u$ are simply transported along the trajectories. So if $x<\tau t$, it lies on a characteristic starting from some point $X<0$. Otherwise if $x>\tau t$, it lies on a characteristic starting from some point $X>0$. Thus, we can conclude that

$$
u(x, t)= \begin{cases}u_{l}, & x<\tau t, \\ u_{r}, & x>\tau t .\end{cases}
$$

Both the shock wave speed $\tau$ and the solution $u(x, t)$ in (29) have no dependence on $\alpha$ such that the limit $\alpha \rightarrow 0$ of the solution $u(x, t)$ in (29) is trivial and not the entropy solution of relativistic Burgers equation (4) due to the fact that they have different shock speeds $\tau \neq \sigma$. The reason lies in the fact that we adopt the averaging of the convective velocity $[24,28]$ (i.e., a Leray-type averaging) for (4) which leads to the different shock speed to the original one. In fact, for a convex scalar conservation law, the averaging of the convective velocity is equivalent to the characteristically averaged method proposed in [21]. Obviously, the Leraytype regularization does not lead to a reasonable result for relativistic Burgers equation (4) and thus it is not an efficient regularization method here.

On the other hand, in the nonrelativistic limit $\varepsilon \rightarrow 0$, it can be deduced from (24) that $\lim _{\varepsilon \rightarrow 0} \tau=\left(u_{l}+u_{r}\right) / 2$. It is easy to check that the limits $\varepsilon \rightarrow 0$ of $\eta(X, t)$ are exactly the trajectories to the Riemann problem for CFB equation (2). Thus, when $u_{l}>u_{r}$, we can see that the Riemann solution for CFRB equation (5) converges to the corresponding one for CFB equation (2) in the nonrelativistic limit $\varepsilon \rightarrow 0$.

Finally, let us compare the different convergence results to the regularization of the convective velocity for relativistic Burgers equation (4) and the Burgers equation (1). For relativistic Burgers equation (4), the shock speed is determined by the Rankine-Hugoniot conditions. While, for CFRB equation (5), the discontinuity speed is determined by the speed of the averaged characteristics at the location of discontinuity. Thus, when $u_{l}>u_{r}$, the Riemann solution for CFRB equation (5) cannot converge to the corresponding one for relativistic Burgers equation (4) due to their different speeds of discontinuities. However, when $u_{l}>u_{r}$, the Riemann solution for CFB equation (2) converges to the corresponding one for Burgers equation (1) due to the fact that they have the same speeds of discontinuities; namely, the equality

$$
\frac{f^{\prime}\left(u_{l}\right)+f^{\prime}\left(u_{r}\right)}{2}=\frac{f\left(u_{r}\right)-f\left(u_{l}\right)}{u_{r}-u_{l}}
$$

holds when $f(u)=u^{2} / 2$. In order to remedy it, we adopt the observable divergence of the vector field to relativistic Burgers equation (4) in the following section.

\section{Riemann Problem for ORB Equation (8)}

In this section, we investigate the Riemann problem for ORB equation (8). In fact, we also restrict ourselves to Helmholtz filter (13) and then look for the exact Riemann solution to (8) and (6) when $u_{l}>u_{r}$. Consequently, we consider the limit $\alpha \rightarrow 0$ of the Riemann solution to (8) and (6) and check that it is exactly the corresponding one for relativistic Burgers equation (4) and (6).

At first, in order to study the weak solutions to ORB equation (8) with the Riemann initial data (6), we should consider the conservative form of (8). Thanks to (18), if we take Helmholtz filter (13), then ORB equation (8) can be rewritten in the following conservative form:

$$
\begin{gathered}
u_{t}+\left(\overline{\left(\frac{u}{\sqrt{1+\varepsilon^{2} u^{2}}+1}\right) \cdot(u-\bar{u})+\frac{u \bar{u}}{\sqrt{1+\varepsilon^{2} u^{2}}+1}}\right. \\
\left.+\alpha^{2} \bar{u}_{x} \cdot \overline{\left(\frac{u}{\sqrt{1+\varepsilon^{2} u^{2}}+1}\right)_{x}}\right)_{x}=0 .
\end{gathered}
$$

We know from [15] that (31) preserves the conservative structure of the original equation (4). It is clear to see that (31) becomes formally relativistic Burgers equation (4) if we take $\alpha=0$ in (31). A natural question is whether solutions of relativistic Burgers equation (4) can be approximated 
properly by those of (31) with the regularization parameter $\alpha>0$, especially for the Riemann initial data (6). In what follows, it will be shown that, for arbitrary $\alpha>0$, the conservative form (31) for the ORB equation (8) has the same traveling wave solution to (4) and (6) when $u_{l}>u_{r}$. Namely, we need to check that solution (10) with (11) to the Riemann problem (4) and (6) is also a weak solution to Riemann problem (31) and (6) as well.

It follows from (13) with (14) that

$$
\begin{aligned}
& \bar{u}(x, t)=\int_{-\infty}^{+\infty} \frac{1}{2 \alpha} \exp \left(-\left|\frac{y}{\alpha}\right|\right) u(x-y, t) d y, \\
& \overline{\left(\frac{u}{\sqrt{1+\varepsilon^{2} u^{2}}+1}\right)}(x, t) \\
& =\int_{-\infty}^{+\infty} \frac{1}{2 \alpha} \exp \left(-\left|\frac{y}{\alpha}\right|\right)\left(\frac{u(x-y, t)}{\sqrt{1+\varepsilon^{2} u(x-y, t)^{2}}+1}\right) d y .
\end{aligned}
$$

By substituting $u(x, t)$ defined as in (10) with (11) into (32), we can obtain that

$$
\begin{aligned}
& \bar{u}(x, t)= \begin{cases}\frac{u_{l}+\frac{u_{r}-u_{l}}{2} \exp \left(\frac{x-\sigma t}{\alpha}\right),}{u_{r}+u_{l}}, & x<\sigma t, \\
u_{r}+\frac{u_{l}-u_{r}}{2} \exp \left(\frac{\sigma t-x}{\alpha}\right), & x>\sigma t,\end{cases} \\
& \overline{\left(\frac{u}{\sqrt{1+\varepsilon^{2} u^{2}}+1}\right)}(x, t) \\
& \left\{\begin{array}{l}
\frac{u_{l}}{\sqrt{1+\varepsilon^{2} u_{l}^{2}}+1} \\
+\frac{1}{2}\left(\frac{u_{r}}{\sqrt{1+\varepsilon^{2} u_{r}^{2}}+1}-\frac{u_{l}}{\sqrt{1+\varepsilon^{2} u_{l}^{2}}+1}\right) \\
\times \exp \left(\frac{x-\sigma t}{\alpha}\right), \\
\frac{1}{2}\left(\frac{u_{r}}{\sqrt{1+\varepsilon^{2} u_{r}^{2}}+1}\right), \\
\left.+\frac{u_{l}}{\sqrt{1+\varepsilon^{2} u_{l}^{2}}+1}\right), \\
\frac{u_{r}}{\sqrt{1+\varepsilon^{2} u_{r}^{2}+1}} \\
+\frac{1}{2}\left(\frac{u_{l}}{\sqrt{1+\varepsilon^{2} u_{l}^{2}}+1}-\frac{x}{\sqrt{1+\varepsilon^{2} u_{r}^{2}}+1}\right.
\end{array}\right),
\end{aligned}
$$

$$
\begin{aligned}
\sigma= & {\left[\overline{\left(\frac{u}{\sqrt{1+\varepsilon^{2} u^{2}}+1}\right) \cdot(u-\bar{u})+\frac{u \bar{u}}{\sqrt{1+\varepsilon^{2} u^{2}}+1}}\right.} \\
& \left.\quad+\alpha^{2} \bar{u}_{x} \cdot \overline{\left(\frac{u}{\sqrt{1+\varepsilon^{2} u^{2}}+1}\right)_{x}}\right] \\
& \times[u]^{-1} .
\end{aligned}
$$

Due to the fact that all the $\bar{u}, \overline{\left(u /\left(\sqrt{1+\varepsilon^{2} u^{2}}+1\right)\right)}, \bar{u}_{x}$, and $\overline{\left(u /\left(\sqrt{1+\varepsilon^{2} u^{2}}+1\right)\right)_{x}}$ are continuous across the discontinuity line $x=\sigma t$, (37) can be reduced to

$$
\begin{aligned}
\sigma= & {\left[\overline{\left(\frac{u}{\sqrt{1+\varepsilon^{2} u^{2}}+1}\right)} \cdot u+\frac{u \bar{u}}{\sqrt{1+\varepsilon^{2} u^{2}}+1}\right] } \\
& \times[u]^{-1}
\end{aligned}
$$

namely

$$
\begin{aligned}
\sigma= & \left(\overline{\left(\frac{u}{\sqrt{1+\varepsilon^{2} u^{2}}+1}\right)}(\sigma t, t) \cdot[u]\right. \\
& \left.+\bar{u}(\sigma t, t) \cdot\left[\frac{u}{\sqrt{1+\varepsilon^{2} u^{2}}+1}\right]\right) \\
& \times[u]^{-1} .
\end{aligned}
$$


Substituting (33) and (34) into (39) yields

$$
\begin{aligned}
\sigma= & \frac{1}{2}\left(\frac{u_{r}}{\sqrt{1+\varepsilon^{2} u_{r}^{2}}+1}+\frac{u_{l}}{\sqrt{1+\varepsilon^{2} u_{l}^{2}}+1}\right) \\
& +\frac{u_{r}+u_{l}}{2\left(\frac{\left.u_{r}-u_{l}\right)}{\sqrt{1+\varepsilon^{2} u_{r}^{2}}+1}-\frac{u_{l}}{\sqrt{1+\varepsilon^{2} u_{l}^{2}}+1}\right)} \\
= & \frac{1}{u_{r}-u_{l}}\left(\frac{u_{r}^{2}}{\sqrt{1+\varepsilon^{2} u_{r}^{2}}+1}-\frac{u_{l}^{2}}{\sqrt{1+\varepsilon^{2} u_{l}^{2}}+1}\right) \\
= & \frac{1}{u_{r}-u_{l}}\left(\frac{\sqrt{1+\varepsilon^{2} u_{r}^{2}}-\sqrt{1+\varepsilon^{2} u_{l}^{2}}}{\varepsilon^{2}}\right) \\
= & \frac{u_{l}+u_{r}}{\sqrt{1+\varepsilon^{2} u_{l}^{2}}+\sqrt{1+\varepsilon^{2} u_{r}^{2}}} .
\end{aligned}
$$

This verifies our claim that, for arbitrary $\alpha>0$, the conservative form (31) for ORB equation (8) has the same traveling wave solution as relativistic Burgers equation (4).

In the following theorem, we will see that $u(x, t)$ defined as in (10) with (11) is indeed the weak solution to Riemann problem (31) and (6).

Theorem 1. Suppose that $u_{l}>u_{r}$, for arbitrary $\alpha>0$; then $u(x, t)$ defined in (10) with (11) is indeed the weak solution the Riemann problem (31) and (6), in which (31) is the conservative form for ORB equation (8) with Helmholtz filter (13).

Proof. In order to prove that $u(x, t)$ defined in (10) with (11) is indeed the weak solution to Riemann problem (31) and (6), let us take a smooth test function $\psi(x, t) \in C_{c}^{\infty}\left(R \times R_{+}\right)$and then check that the equality

$$
\begin{aligned}
\left\langle u_{t}+\left(\overline{\left(\frac{u}{\sqrt{1+\varepsilon^{2} u^{2}}+1}\right)} \cdot(u-\bar{u})\right.\right. \\
+\frac{u \bar{u}}{\sqrt{1+\varepsilon^{2} u^{2}}+1}+\alpha^{2} \bar{u}_{x} \\
\left.\left.\quad \cdot\left(\frac{u}{\sqrt{1+\varepsilon^{2} u^{2}}+1}\right)_{x}\right)_{x}, \psi(x, t)\right\rangle=0
\end{aligned}
$$

holds in the weak (or distribution) sense. To prove the above claim, we need to compute each term on the left hand of (41) and consequently verify that their sum is zero. At first, we have

$$
\begin{aligned}
\left\langle u_{t}, \psi\right\rangle= & -\int_{0}^{\infty} \int_{-\infty}^{\infty} u(x, t) \psi_{t}(x, t) d x d t \\
= & -\int_{0}^{\infty} \int_{-\infty}^{\sigma t} u_{l} \psi_{t}(x, t) d x d t \\
& -\int_{0}^{\infty} \int_{\sigma t}^{\infty} u_{r} \psi_{t}(x, t) d x d t .
\end{aligned}
$$

If $\sigma>0$, by exchanging the ordering of integration, then we have

$$
\begin{aligned}
\left\langle u_{t}, \psi\right\rangle= & -\int_{-\infty}^{0} \int_{0}^{\infty} u_{l} \psi_{t}(x, t) d t d x \\
& -\int_{0}^{\infty} \int_{x / \sigma}^{\infty} u_{l} \psi_{t}(x, t) d t d x \\
& -\int_{0}^{\infty} \int_{0}^{x / \sigma} u_{r} \psi_{t}(x, t) d x d t \\
= & \int_{0}^{\infty}\left(u_{l}-u_{r}\right) \psi\left(x, \frac{x}{\sigma}\right) d x \\
= & \int_{0}^{\infty} \sigma\left(u_{l}-u_{r}\right) \psi(\sigma t, t) d t
\end{aligned}
$$

in which we have used the change of variables and the fact that $\psi$ is compact support. Similarly, if $\sigma<0$, then we also have

$$
\begin{aligned}
\left\langle u_{t}, \psi\right\rangle= & -\int_{-\infty}^{0} \int_{0}^{x / \sigma} u_{l} \psi_{t}(x, t) d t d x \\
& -\int_{-\infty}^{0} \int_{x / \sigma}^{\infty} u_{r} \psi_{t}(x, t) d t d x \\
& -\int_{0}^{\infty} \int_{0}^{\infty} u_{r} \psi_{t}(x, t) d x d t \\
= & \int_{-\infty}^{0}\left(u_{r}-u_{l}\right) \psi\left(x, \frac{x}{\sigma}\right) d x \\
= & \int_{0}^{\infty} \sigma\left(u_{l}-u_{r}\right) \psi(\sigma t, t) d t
\end{aligned}
$$

Secondly, we deduce that

$$
\begin{aligned}
& \left\langle\left(\overline{\left(\frac{u}{\sqrt{1+\varepsilon^{2} u^{2}}+1}\right)} \cdot(u-\bar{u})\right)_{x}, \psi\right\rangle \\
& =-\int_{0}^{\infty} \int_{-\infty}^{\infty} \overline{\left(\frac{u}{\sqrt{1+\varepsilon^{2} u^{2}}+1}\right)} \cdot(u-\bar{u}) \psi_{x}(x, t) d x d t \\
& =-\int_{0}^{\infty} \int_{-\infty}^{\sigma t}\left(\frac{u_{l}}{\sqrt{1+\varepsilon^{2} u_{l}^{2}}+1}\right. \\
& +\frac{1}{2}\left(\frac{u_{r}}{\sqrt{1+\varepsilon^{2} u_{r}^{2}}+1}-\frac{u_{l}}{\sqrt{1+\varepsilon^{2} u_{l}^{2}}+1}\right) \\
& \left.\times \exp \left(\frac{x-\sigma t}{\alpha}\right)\right) \\
& \times \frac{u_{l}-u_{r}}{2} \exp \left(\frac{x-\sigma t}{\alpha}\right) \psi_{x}(x, t) d x d t
\end{aligned}
$$




$$
\begin{aligned}
-\int_{0}^{\infty} \int_{\sigma t}^{\infty}( & \frac{u_{r}}{\sqrt{1+\varepsilon^{2} u_{r}^{2}}+1} \\
& +\frac{1}{2}\left(\frac{u_{l}}{\sqrt{1+\varepsilon^{2} u_{l}^{2}}+1}-\frac{u_{r}}{\sqrt{1+\varepsilon^{2} u_{r}^{2}}+1}\right) \\
& \left.\times \exp \left(\frac{\sigma t-x}{\alpha}\right)\right) \\
& \times \frac{u_{r}-u_{l}}{2} \exp \left(\frac{\sigma t-x}{\alpha}\right) \psi_{x}(x, t) d x d t
\end{aligned}
$$

in which (10), (33), and (34) have been used.

Thirdly, in view of (10) and (33) again, we have

$$
\begin{aligned}
& \left\langle\left(\frac{u \bar{u}}{\sqrt{1+\varepsilon^{2} u^{2}}+1}\right)_{x}, \psi\right\rangle \\
& =-\int_{0}^{\infty} \int_{-\infty}^{\infty}\left(\frac{u \bar{u}}{\sqrt{1+\varepsilon^{2} u^{2}}+1}\right) \psi_{x}(x, t) d x d t \\
& =-\int_{0}^{\infty} \int_{-\infty}^{\sigma t}\left(\frac{u_{l}}{\sqrt{1+\varepsilon^{2} u_{l}^{2}}+1}\right) \\
& \quad \times\left(\frac{\left.u_{l}+\frac{u_{r}-u_{l}}{2} \exp \left(\frac{x-\sigma t}{\alpha}\right)\right) \psi_{x}(x, t) d x d t}{-\int_{0}^{\infty} \int_{\sigma t}^{\infty}\left(\frac{u_{r}}{\sqrt{1+\varepsilon^{2} u_{r}^{2}}+1}\right)}\right. \\
& \times\left(u_{r}+\frac{u_{l}-u_{r}}{2} \exp \left(\frac{\sigma t-x}{\alpha}\right)\right) \psi_{x}(x, t) d x d t .
\end{aligned}
$$

Finally, by virtue of (35) and (36), we arrive at

$$
\begin{gathered}
\left\langle\left(\alpha^{2} \bar{u}_{x} \cdot \overline{\left.\left.\left(\frac{u}{\sqrt{1+\varepsilon^{2} u^{2}}+1}\right)_{x}\right)_{x}, \psi\right\rangle}\right.\right. \\
=-\int_{0}^{\infty} \int_{-\infty}^{\infty}\left(\alpha^{2} \bar{u}_{x} \cdot\left(\frac{u}{\sqrt{1+\varepsilon^{2} u^{2}}+1}\right)_{x}\right) \psi_{x}(x, t) d x d t \\
=-\int_{0}^{\infty} \int_{-\infty}^{\sigma t}\left(\frac{u_{r}-u_{l}}{4}\right)\left(\frac{u_{r}}{\sqrt{1+\varepsilon^{2} u_{r}^{2}}+1}-\frac{u_{l}}{\sqrt{1+\varepsilon^{2} u_{l}^{2}}+1}\right) \\
\quad \times \exp \left(\frac{2(x-\sigma t)}{\alpha}\right) \psi_{x}(x, t) d x d t
\end{gathered}
$$

$$
\begin{aligned}
-\int_{0}^{\infty} \int_{\sigma t}^{\infty}\left(\frac{u_{r}-u_{l}}{4}\right)\left(\frac{u_{r}}{\sqrt{1+\varepsilon^{2} u_{r}^{2}}+1}-\frac{u_{l}}{\sqrt{1+\varepsilon^{2} u_{l}^{2}}+1}\right) \\
\quad \times \exp \left(\frac{2(\sigma t-x)}{\alpha}\right) \psi_{x}(x, t) d x d t
\end{aligned}
$$

Collecting (45), (46), and (47) together, one can see that

$$
\begin{aligned}
& \left\langle\overline{\left(\frac{u}{\sqrt{1+\varepsilon^{2} u^{2}}+1}\right)} \cdot(u-\bar{u})+\frac{u \bar{u}}{\sqrt{1+\varepsilon^{2} u^{2}}+1}\right. \\
& \left.\left.+\alpha^{2} \bar{u}_{x} \cdot \overline{\left(\frac{u}{\sqrt{1+\varepsilon^{2} u^{2}}+1}\right)_{x}}\right)_{x}, \psi(x, t)\right\rangle \\
& =-\int_{0}^{\infty} \int_{-\infty}^{\sigma t} \frac{u_{l}^{2}}{\sqrt{1+\varepsilon^{2} u_{l}^{2}}+1} \cdot \psi_{x}(x, t) d x d t \\
& -\int_{0}^{\infty} \int_{\sigma t}^{\infty} \frac{u_{r}^{2}}{\sqrt{1+\varepsilon^{2} u_{r}^{2}}+1} \cdot \psi_{x}(x, t) d x d t \\
& =\int_{0}^{\infty}\left(\frac{u_{r}^{2}}{\sqrt{1+\varepsilon^{2} u_{r}^{2}}+1}-\frac{u_{l}^{2}}{\sqrt{1+\varepsilon^{2} u_{l}^{2}}+1}\right) \cdot \psi(\sigma t, t) d t \\
& =\int_{0}^{\infty} \sigma\left(u_{r}-u_{l}\right) \psi(\sigma t, t) d t
\end{aligned}
$$

in which we have used the result in (40). Thus, it is clear that the addition of (48) and (43) for $\sigma>0$ (or (48) and (44) for $\sigma<0)$ leads to equality (41).

It can be drawn from the above theorem that $u(x, t)$ defined in (10) with (11) is indeed the weak solution to Riemann problem (31) and (6), which implies that it has no dependence on the regularization parameter $\alpha$. It is clear to see that that the Riemann solution to (31) and (6) converges to the corresponding one to (4) and (6) as $\alpha \rightarrow 0$, which is the desirable result. It is remarkable that the shock position is invariant for Riemann problem (31) and (6) during the limit process $\alpha \rightarrow 0$. Thus, the observable divergence method is reasonable to capture the shock solution for relativistic Burgers equation (4).

\section{Conclusions}

From the above results, we can see that a Leray-type averaging, namely, an averaging of the convective velocity, is successful to regularize Burgers equation (1) due to the fact that (1) obeys special condition (30). Thus, this averaging cannot be extended to the general scalar conservation laws, not to mention systems of hyperbolic conservation laws. Here we take relativistic Burgers equation (4) as an example to explain it.

In order to remedy it, Mohseni has developed the observable divergence method to study the shock regularization for 
systems of hyperbolic conservation laws. In this paper, we take relativistic Burgers equation (4) as the detailed example to check and confirm that it is an efficient regularization technique due to the fact that it preserves the shock speed of the original equation. In particular, we use the Helmholtz filter such that regularized system (8) can be still written in conservative form (31), and thus it preserves the original conservation properties of relativistic Burgers equation (4). It is clear to see that the results in this paper can be extended to the convex (or concave) scalar conservation law. Furthermore, compared with a Leray-type averaging, the observable divergence method may be more appropriately extended to systems of conservation laws.

\section{Conflict of Interests}

The authors declare that there is no conflict of interests regarding the publication of this paper.

\section{Acknowledgments}

This work is partially supported by the National Natural Science Foundation of China $(11001116,11271176)$ and the Project of Shandong Provincial Higher Educational Science and Technology Program (J12LI01, J11LA03).

\section{References}

[1] S. Chen, C. Foias, D. D. Holm, E. Olson, E. S. Titi, and S. Wynne, "Camassa-Holm equations as a closure model for turbulent channel and pipe flow," Physical Review Letters, vol. 81, no. 24, pp. 5338-5341, 1998.

[2] S. Chen, C. Foias, D. D. Holm, E. Olson, E. S. Titi, and S. Wynne, "The Camassa-Holm equations and turbulence," Physica D: Nonlinear Phenomena, vol. 133, no. 1-4, pp. 49-65, 1999.

[3] S. Chen, C. Foias, D. D. Holm, E. Olson, E. S. Titi, and S. Wynne, "A connection between the Camassa-Holm equations and turbulent flows in channels and pipes," Physics of Fluids, vol. 11, no. 8, pp. 2343-2353, 1999.

[4] A. Cheskidov, D. D. Holm, and E. a. . Olson, "On a Leray- $\alpha$ model of turbulence," Proceedings of the Royal Society of London A, vol. 461, no. 2055, pp. 629-649, 2005.

[5] J. Leray, "Sur le mouvement d'un liquide visqueux emplissant l'espace," Acta Mathematica, vol. 63, no. 1, pp. 193-248, 1934.

[6] H. Ali, "On a critical Leray- $\alpha$ model of turbulence," Nonlinear Analysis: Real World Applications, vol. 14, no. 3, pp. 1563-1584, 2013.

[7] C. Cao, D. D. Holm, and E. S. Titi, "Traveling wave solutions for a class of one-dimensional nonlinear shallow water wave models," Journal of Dynamics and Differential Equations, vol. 16, no. 1, pp. 167-178, 2004.

[8] Y. Cao, E. M. Lunasin, and E. S. Titi, "Global well-posedness of the three-dimensional viscous and inviscid simplified Bardina turbulence models," Communications in Mathematical Sciences, vol. 4, no. 4, pp. 823-848, 2006.

[9] C. Foias, D. D. Holm, and E. S. Titi, “The Navier-Stokes-alpha model of fluid turbulence," Physica D: Nonlinear Phenomena, vol. 152-153, pp. 505-519, 2001.

[10] C. Foias, D. D. Holm, and E. S. Titi, "The three dimensional viscous Camassa-Holm equations, and their relation to the Navier-Stokes equations and turbulence theory," Journal of Dynamics and Differential Equations, vol. 14, no. 1, pp. 1-35, 2002.

[11] A. A. Ilyin, E. M. Lunasin, and E. S. Titi, "A modified-Leray- $\alpha$ subgrid scale model of turbulence," Nonlinearity, vol. 19, no. 4, pp. 879-897, 2006.

[12] A. Larios and E. S. Titi, "On the higher-order global regularity of the inviscid Voigt-regularization of three-dimensional hydrodynamic models," Discrete and Continuous Dynamical Systems. Series B, vol. 14, no. 2, pp. 603-627, 2010.

[13] A. Larios and E. S. Titi, "Higher-order global regularity of an inviscid Voigt-regularization of the three-dimensional inviscid resistive magnetohydrodynamic equations," Journal of Mathematical Fluid Mechanics, vol. 16, no. 1, pp. 59-76, 2014.

[14] G. Norgard and K. Mohseni, "A regularization of the Burgers equation using a filtered convective velocity," Journal of Physics A: Mathematical and Theoretical, vol. 41, pp. 1-21, 2008.

[15] G. Norgard and K. Mohseni, "A new potential regularization of the one-dimensional Euler and homentropic Euler equations," Multiscale Modeling \& Simulation, vol. 8, no. 4, pp. 1212-1243, 2010.

[16] L. C. Berselli and L. Bisconti, "On the structural stability of the Euler-Voigt and Navier-Stokes-Voigt models," Nonlinear Analysis. Theory, Methods \& Applications, vol. 75, no. 1, pp. 117130, 2012.

[17] L. C. Berselli and R. Lewandowski, "Convergence of approximate deconvolution models to the mean Navier-Stokes equations," Annales de l'Institut Henri Poincare (C): Non Linear Analysis, vol. 29, no. 2, pp. 171-198, 2012.

[18] B. Dong, G. Sadek, and Z. Chen, "On the regularity criteria of the 3D Navier-Stokes equations in critical spaces," Acta Mathematica Scientia B (English Edition), vol. 31, no. 2, pp. 591600, 2011.

[19] E. Olson and E. S. Titi, "Viscosity versus vorticity stretching: global well-posedness for a family of Navier-Stokes-alpha-like models," Nonlinear Analysis: Theory, Methods \& Applications, vol. 66, no. 11, pp. 2427-2458, 2007.

[20] J. E. Marsden and S. Shkoller, "Global well-posedness for the Lagrangian averaged Navier-Stokes (LANS- $\alpha$ ) equations on bounded domains," The Royal Society of London A, vol. 359, no. 1784, pp. 1449-1468, 2001.

[21] G. J. Norgard and K. Mohseni, "An examination of the homentropic Euler equations with averaged characteristics," Journal of Differential Equations, vol. 248, no. 3, pp. 574-593, 2010.

[22] C. Shen, "On a regularization of a scalar conservation law with discontinuous coefficients," Journal of Mathematical Physics, vol. 55, no. 3, Article ID 031502, 15 pages, 2014.

[23] J. Villavert and K. Mohseni, "An inviscid regularization of hyperbolic conservation laws," Journal of Applied Mathematics and Computing, vol. 43, no. 1-2, pp. 55-73, 2013.

[24] F. Weber, Conservation laws with convectively filtered variables [Master thesis], Swiss Federal Institute of Technology, Zurich, Switzerland, 2012.

[25] E. Hopf, "The partial differential equation $u_{t}+u u_{x}=\mu_{x x}$ ", Communications on Pure and Applied Mathematics, vol. 3, pp. 201-230, 1950

[26] P. D. Lax and C. D. Levermore, "The small dispersion limit of the Korteweg-de Vries equation.II," Communications on Pure and Applied Mathematics, vol. 36, no. 5, pp. 571-593, 1983.

[27] K. Mohseni, H. Zhao, and J. E. Marsden, "Shock regularization for the Burgers equation," in Proceedings of the 44th AIAA Aerospace Sciences Meeting, pp. 18074-18080, January 2006. 
[28] H. S. Bhat and R. C. Fetecau, "The Riemann problem for the Leray-Burgers equation," Journal of Differential Equations, vol. 246, no. 10, pp. 3957-3979, 2009.

[29] H. S. Bhat and R. C. Fetecau, "Stability of fronts for a regularization of the Burgers equation," Quarterly of Applied Mathematics, vol. 66, no. 3, pp. 473-496, 2008.

[30] G. Norgard and K. Mohseni, "On the convergence of the convectively filtered Burgers equation to the entropy solution of the inviscid Burgers equation," Multiscale Modeling \& Simulation, vol. 7, no. 4, pp. 1811-1837, 2009.

[31] P. G. LeFloch, H. Makhlof, and B. Okutmustur, "Relativistic Burgers equations on curved spacetimes: derivation and finite volume approximation," SIAM Journal on Numerical Analysis, vol. 50, no. 4, pp. 2136-2158, 2012.

[32] K. Mohseni, "Derivation and numerical simulation of regularized (observable) Euler equations," in Proceedings of the 48th AIAA Aerospace Sciences Meeting Including the New Horizons Forum and Aerospace Exposition, AIAA Paper 2010-1274, Orlando, Fla, USA, January 2010.

[33] J. Bardina, J. H. Ferziger, and W. C. Reynold, "Improved turbulence models based on large eddy simulation of homogemeous, incompressible, turbulent flows," Tech. Rep. TF-19, Department of Mechanical Engineering, Standford University, Standford, Calif, USA, 1983.

[34] W. Layton and R. Lewandowski, "On a well-posed turbulence model," Discrete and Continuous Dynamical Systems B: A Journal Bridging Mathematics and Sciences, vol. 6, no. 1, pp. 111128, 2006.

[35] S. Stolz and N. A. Adams, "An approximate deconvolution procedure for large-eddy simulation," Physics of Fluids, vol. 11, no. 7, pp. 1699-1701, 1999.

[36] J. D. Gibbon and D. D. Holm, "Estimates for the LANS- $\alpha$, Leray$\alpha$ and Bardina models in terms of a Navier-Stokes Reynolds number," Indiana University Mathematics Journal, vol. 57, no. 6, pp. 2761-2773, 2008.

[37] R. Lewandowski, "On Bardina and approximate deconvolution models," Séminaire Laurent Schwartz-EDP et Applications (2011-2012), pp. 1-12, 2012. 


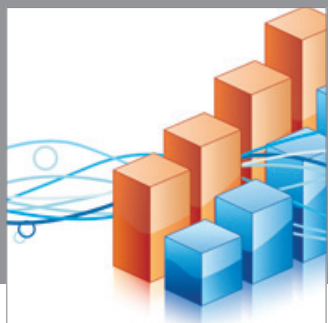

Advances in

Operations Research

mansans

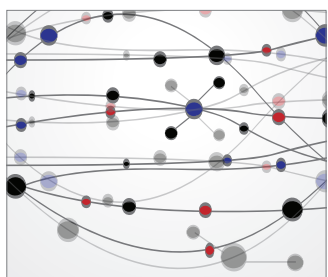

The Scientific World Journal
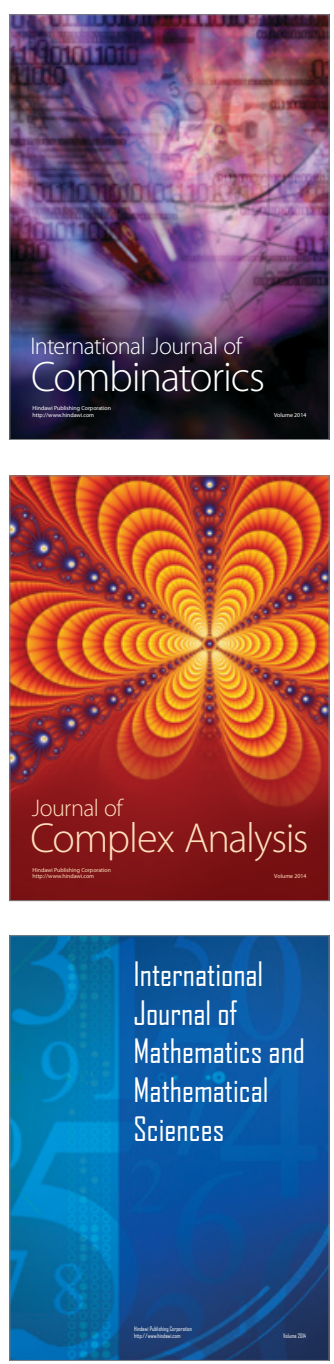
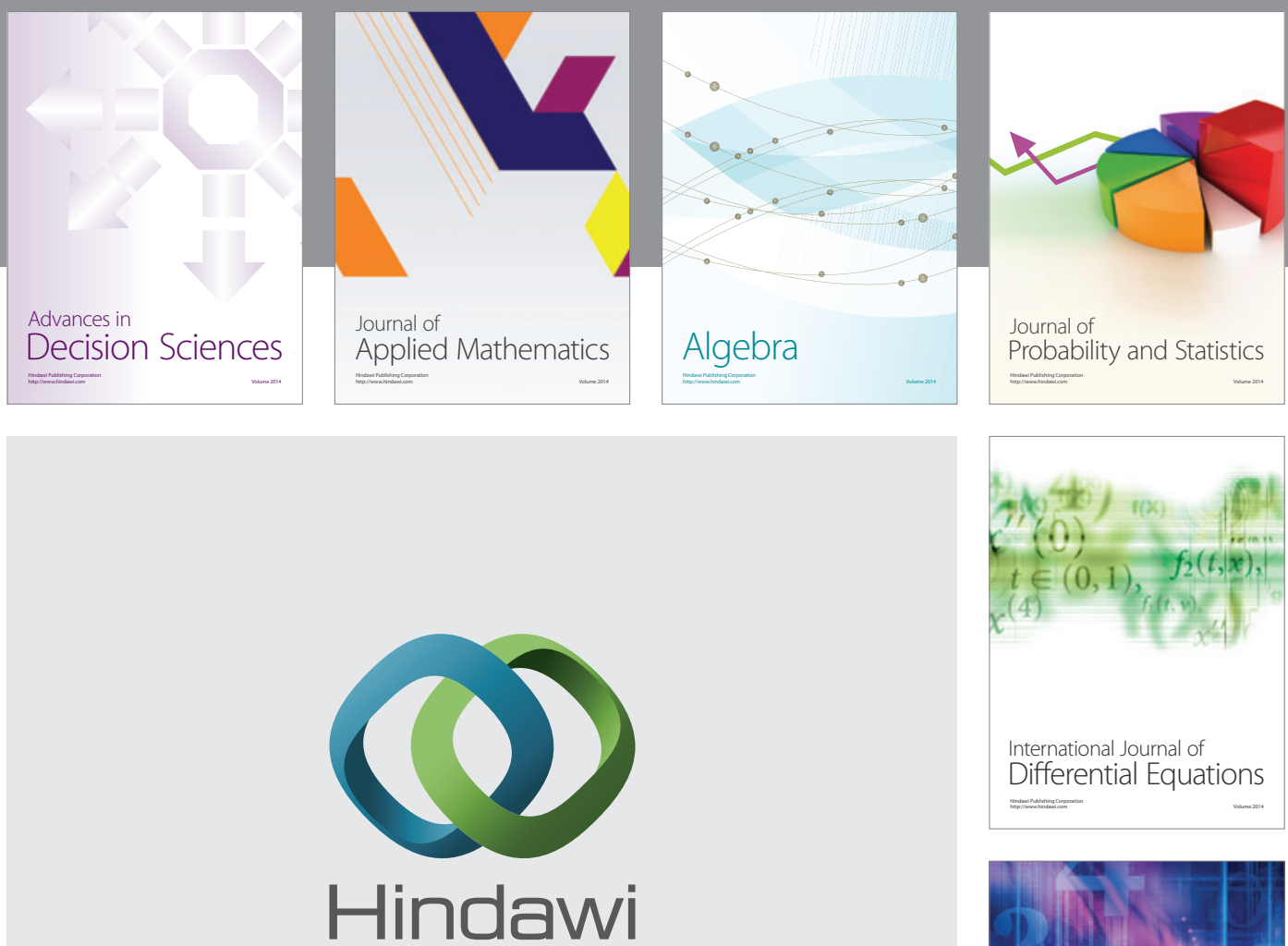

Submit your manuscripts at http://www.hindawi.com
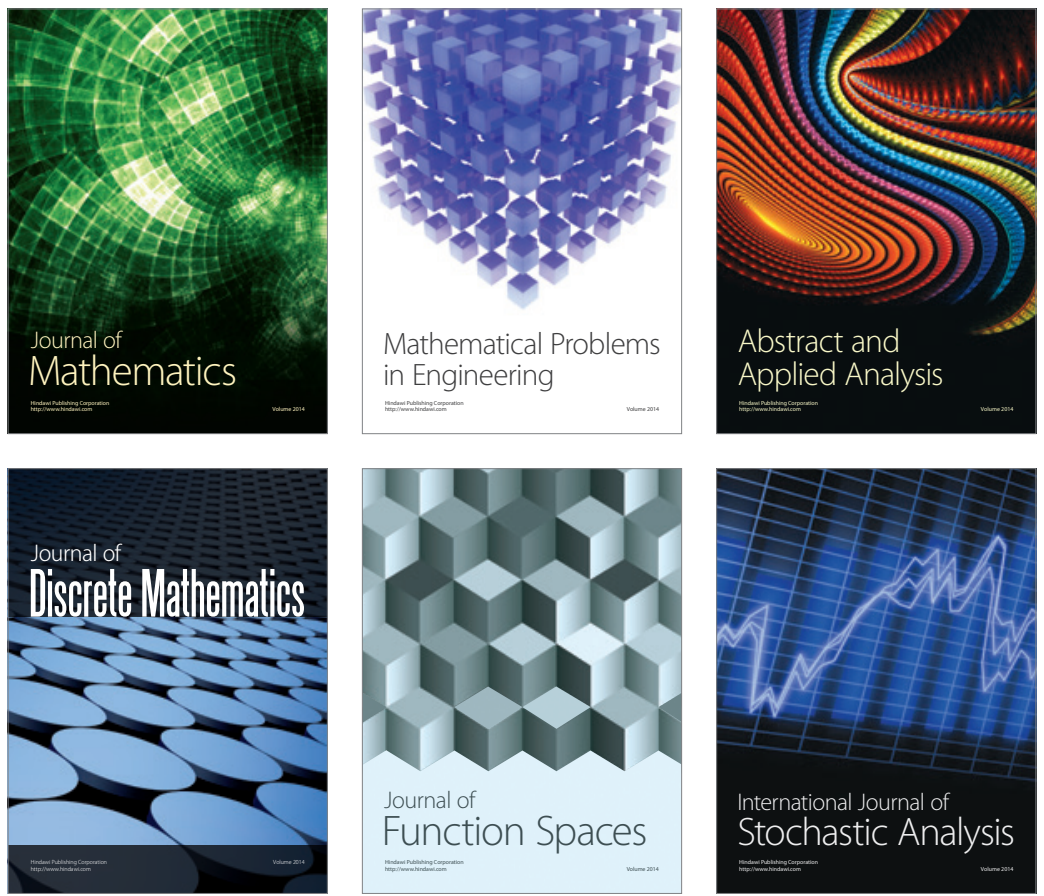

Journal of

Function Spaces

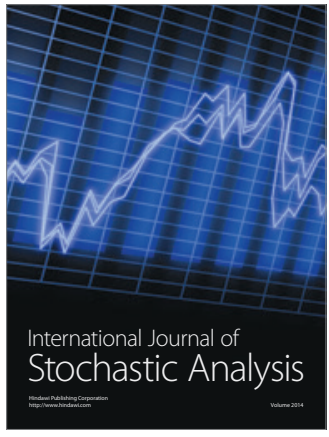

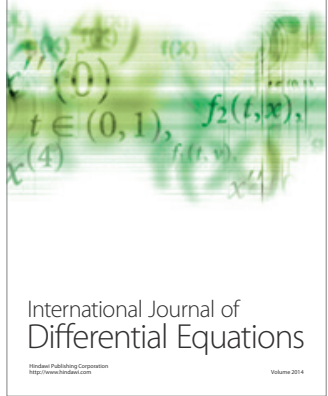
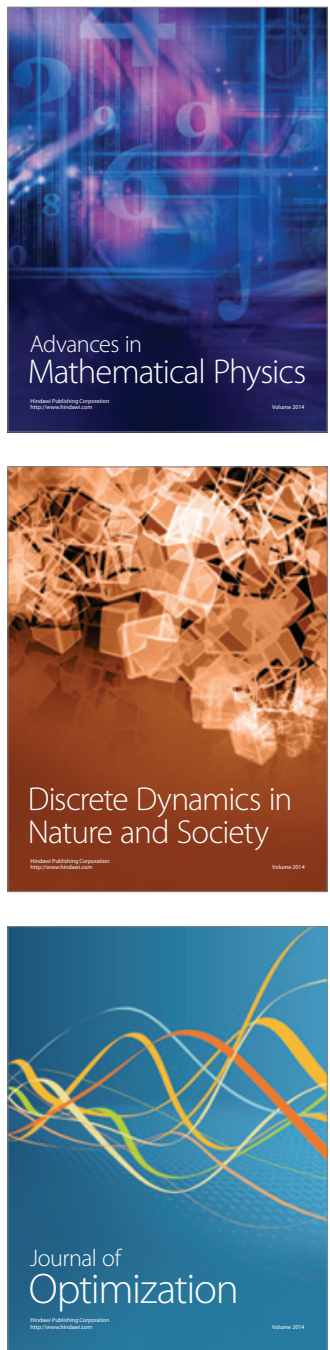